\title{
NONLINEAR ACTUATOR FAULT ESTIMATION OBSERVER: AN INVERSE SYSTEM APPROACH VIA A T-S FUZZY MODEL
}

\author{
DEZHI XU*, BIN JIANG*, PENG SHI **,*** \\ * College of Automation Engineering \\ Nanjing University of Aeronautics and Astronautics, Nanjing 210016, China \\ e-mail: binjiangenuaa. edu. cn \\ ** Department of Computing and Mathematical Sciences \\ University of Glamorgan, Pontypridd CF37 1DL, UK \\ ${ }^{* * *}$ School of Engineering and Science \\ Victoria University, Melbourne, Vic 8001, Australia \\ e-mail: peng.shi@vu.edu.au
}

\begin{abstract}
Based on a Takagi-Sugeno (T-S) fuzzy model and an inverse system method, this paper deals with the problem of actuator fault estimation for a class of nonlinear dynamic systems. Two different estimation strategies are developed. Firstly, T-S fuzzy models are used to describe nonlinear dynamic systems with an actuator fault. Then, a robust sliding mode observer is designed based on a T-S fuzzy model, and an inverse system method is used to estimate the actuator fault. Next, the second fault estimation strategy is developed. Compared with some existing techniques, such as adaptive and sliding mode methods, the one presented in this paper is easier to be implemented in practice. Finally, two numerical examples are given to demonstrate the efficiency of the proposed techniques.
\end{abstract}

Keywords: actuator fault estimation, Takagi-Sugeno fuzzy models, robust sliding mode observer, inverse system method.

\section{Introduction}

To improve system performance efficiency, maintainability and reliability can be achieved by designing FaultTolerant Control (FTC), which relies on early detection of fault, using Fault Detection and Isolation (FDI) procedures, and on fault accommodation or system reconfiguration strategies, to achieve the system goal in spite of the faults. The fault-tolerant design approach can be mainly classified into two types: passive and active (Staroswiecki and Gehin, 2001). In the passive approach, the same controller is used throughout the normal case as well as the fault case such that this passive fault-tolerant controller can be easily implemented ( $\mathrm{Gu}$ et al., 2010; Pang and Tang, 2010). An active FTC system compensates for the effect of the fault by synthesizing a new control strategy based on online accommodation (Xu et al., 2011a; Guo et al., 2010).

System reconfiguration is the strategy with which the goals are achieved by switching off the faulty part of the system and controlling only its healthy part (Staroswiecki and Gehin, 2001). For system reconfiguration, FDI algorithms should only detect and isolate the faults (Shumsky, 2007). The design and analysis of such algorithms have received considerable attention during the past two decades. Fruitful results can be found in several excellent survey papers (Zhang and Jiang, 2008; Isermann, 2005) and books (Chen and Patton, 1999; Vachtsevanos et al., 2006; Isermann, 2006).

Most research work on FDI has concentrated on linear systems and only limited results for nonlinear systems have been reported. Early work on fault diagnosis for a class of nonlinear systems was investigated by Seliger and Frank (1991) using the unknown input observer approach (Guan and Saif, 1991), while recently some results on FDI for nonlinear systems have been obtained, e.g., by Edwards et al. (2000), Yan and Edwards (2007), or Jiang et al. (2006) based on nonlinear observers, Staroswiecki and Gehin (2001) based on parity space approaches, and Christophe et al. (2002) exploiting the relationship between the two methods. More recently, Persis and Isidori 
(2001) investigated the problem of fault detection and isolation for nonlinear systems using a differential geometric approach. Observer design was dealt with by Edwards et al. (2000) to maintain a sliding motion even in the presence of faults which are detected by analysing the socalled equivalent output injection.

Fault accommodation is the strategy with which the goals are achieved by controlling the fault system (Staroswiecki and Gehin, 2001), which means that only the controller is reconfigured. If a fault is detected and isolated, the fault also needs to be estimated so that its effect can be compensated by adapting (reconfiguring) the control algorithm. Compared with FDI only, fault estimation is not an easy task. However, some results for fault diagnosis/estimation have been obtained based on adaptive observers (Ding and Frank, 1993; Jiang et al., 2001; Jiang et al., 2010) unknown input observers (Fu et al., 2004) and using a learning approach (Polycarpou, 2001). But uncertainty exists in the model of an actual plant, so fault estimation for uncertain systems has been studied. Jiang et al. (2006) applied robust/sliding-mode observers to estimate the faults for an affine nonlinear system with uncertainty. Yan and Edwards (2007) used sliding-mode observers to estimate faults, but the estimation error depends on the bounds on the uncertainty.

In recent years, there has been a growing interest in the Takagi-Sugeno (T-S) fuzzy modeling technique since it is a powerful solution that bridges the gap between linear and nonlinear control systems (Zhang and Jiang, 2010; Jiang et al., 2010; Gao et al., 2010; Nguang et al., 2007; Wu et al., 2011). The important advantage of a T-S fuzzy system is its universal approximation of any smooth nonlinear function by mixing some local linear system models. This greatly facilitates the analysis and synthesis of complex nonlinear systems. Many important results on the analysis and synthesis for a T-S fuzzy system have been reported (cf. Zhou et al., 2007; Nguang and Shi, 2003; Lendek et al., 2010a; Gao et al., 2009; Takagi and Sugeno, 1985; Boukezzoula et al., 2003). Because of the advantage of T-S fuzzy systems in approximating complex nonlinear systems, the fault diagnosis observer based on a T-S fuzzy system was investigated using adaptive observers (Jiang et al., 2010; Gao et al., 2010; Lendek et al., 2010a; Lendek et al., 2010b; Zhang and Jiang, 2010) or unknown input observers (Lendek et al., 2010a; Lendek et al., 2010b; Chen and Saif, 2010).

Based on the aforementioned works, this paper develops a methodology for comprehensive estimation algorithm of actuator faults in nonlinear systems. The T-S fuzzy modeling technique is firstly employed to approximate the nonlinear dynamic system, and an fault model is developed. Then, two different actuator fault estimation strategies are proposed. For the first strategy, a T-S fuzzy observer has been designed based on the T-S fuzzy model and a sliding mode technique, which is used to observe the state for the nonlinear system. The designed filter estimates a high-order derivative of the output. Next, using the inverse system of the nonlinear system, the actuator fault can be estimated. The second strategy, combining a $\mathrm{T}-\mathrm{S}$ fuzzy observer and the inverse system, is used to estimate and observe the actuator fault and state. It does not have to use adaptive and sliding mode techniques. Apart from that the paper not only outlines estimation of actuator faults, but also provides the state observer. The estimated actuator fault inputs could be potentially employed for the development of a fault tolerant control system. Finally, simulation results are presented to demonstrate the effectiveness of the proposed strategies.

\section{Problem formulation and preliminaries}

Consider the nonlinear invertible system

$$
\begin{aligned}
& \dot{x}(t)=f\left(x(t), u(t), d(t), f_{a}(t)\right), \\
& y(t)=h(x(t))
\end{aligned}
$$

where $x(t) \in \mathbb{R}^{r}$ is the state vector, $u(t) \in \mathbb{R}^{m}$ is the input vector, $y(t) \in \mathbb{R}^{n}$ is the output vector, $f_{a}(t) \in \mathbb{R}^{m}$ is the actuator fault signal, $d(t) \in \mathbb{R}^{p}$ is the unmeasurable disturbance, and $f(\cdot)$ and $h(\cdot)$ are nonlinear functions. The continuous actuator fault is modeled by a "fault pattern" as in Zhang and Jiang (2010), Yang (2004), as well as Patton et al. (2001). The "fault signal" $f_{a}(t)<f_{a_{M}}$ represents the unexpected disturbance in the control channel and can be constant or time varying.

Assume the system is locally observable that and the disturbance $d(t)$ can be identified. According to input equivalent disturbance (LED) of Xie et al. (1999), the system (1) is re-written in the following form:

$$
\begin{aligned}
& \dot{x}(t)=f(x(t),(u(t)+\mu(t))), \\
& y(t)=h(x(t)) .
\end{aligned}
$$

This model lumps an actuator fault, the time-varying parameter, unmeasurable disturbance and unmodeled dynamics into $\mu(t)$.

Nonlinear systems can be approximated as locally linear systems is much the same way as nonlinear functions can be approximated as piecewise linear functions. Systems (2) can be represented by T-S fuzzy models of the following forms: THEN

$$
\text { IF } z_{1}(t) \text { is } M_{1}^{i}, z_{2}(t) \text { is } M_{2}^{i} \text { and } \cdots \cdots z_{q}(t) \text { is } M_{q}^{i} \text {, }
$$

$$
\begin{gathered}
\dot{x}(t)=A_{i} x(t)+B_{i} u(t)+B_{i} \mu(t), \\
y(t)=C_{i} x(t), \quad i=1, \ldots, L .
\end{gathered}
$$

This is referred to as a Takagi-Sugeno model. The quantities $z(t)=\left[z_{1}(t) z_{2}(t) \ldots z_{q}(t)\right]$ are the premise variables, and $M_{1}^{i}, \ldots, M_{q}^{i}$ are fuzzy sets. $A_{i}, B_{i}$ and $C_{i}$ 
are known real constant matrices with appropriate dimensions. Each of the $L$ local models of (3) and (4) is a linear time-invariant model. A fuzzy combination of these local models results in the global model

$$
\begin{aligned}
& \dot{x}(t)=\sum_{i=1}^{L} h_{i}(z(t))\left[A_{i} x(t)+B_{i} u(t)+B_{i} \mu(t)\right], \\
& y(t)=\sum_{i=1}^{L} h_{i}(z(t)) C_{i} x(t),
\end{aligned}
$$

where the membership grades $h_{i}(z(t))$ are defined as

$$
\begin{aligned}
& h_{i}(z(t))=\frac{\nu_{i}(z(t))}{\sum_{i=1}^{L} \nu_{i}(z(t))}, \\
& \nu_{i}(z(t))=\prod_{j=1}^{q} M_{j}^{i}(z(t)) .
\end{aligned}
$$

Hence, $h_{i}(z)$ satisfies the following conditions:

$$
h_{i}(z(t)) \in[0,1], \quad \sum_{i=1}^{L} h_{i}(z(t))=1 .
$$

From (5) and (6) we can derive

$$
\begin{aligned}
& \dot{x}(t)=A(t) x(t)+B(t) u(t)+B(t) \mu(t), \\
& y(t)=C(t) x(t),
\end{aligned}
$$

where $A(t), B(t)$ and $C(t)$ are given as

$$
\begin{aligned}
& A(t)=\sum_{i=1}^{L} h_{i}(z(t)) A_{i}, \\
& B(t)=\sum_{i=1}^{L} h_{i}(z(t)) B_{i}, \\
& C(t)=\sum_{i=1}^{L} h_{i}(z(t)) C_{i} .
\end{aligned}
$$

In other words, the global model, which is a fuzzy combination of $L$ local linear time invariant models, can be represented as a time-varying model. If the premise variables $z(t)$ are functions of the state or control, then the model is nonlinear. However, if the premise variables are independent of the state or control, then the model is linear.

Now we define $L$ continuous time signals $x_{i}(t)$ and $L$ continuous time signals $y_{i}(t)$ as

$$
x_{i}(t)=h_{i}(z(t)) x(t), \quad y_{i}(t)=h_{i}(z(t)) y(t) .
$$

From these definitions and (8), it can be seen that

$$
x(t)=\sum_{i=1}^{L} x_{i}(t), \quad y(t)=\sum_{i=1}^{L} y_{i}(t) .
$$

The dynamic behavior of $x_{i}(k)$ and $y_{i}(k)$ is presented in the following lemma.
Lemma 1. The T-S fuzzy model (5) and (6) can be described as follows:

$$
\begin{aligned}
\dot{x}_{i}(t)= & A_{i} x_{i}(t)+h_{i}(z(t)) B_{i} u(t) \\
& +h_{i}(z(t)) B_{i} \mu(t), \\
y_{i}(t)= & C_{i} x_{i}(t), \quad i=1, \ldots, L .
\end{aligned}
$$

Proof. From (12)-(14), we obtain

$$
\begin{aligned}
\dot{x}(t)= & \sum_{i=1}^{L} \dot{x}_{i}(t) \\
= & \sum_{i=1}^{L}\left[A_{i} x_{i}(t)+h_{i}(z(t)) B_{i} u(t)+h_{i}(z(t)) B_{i} \mu(t)\right] \\
= & \sum_{i=1}^{L} A_{i} h_{i}(z(t)) x(t)+\sum_{i=1}^{L} h_{i}(z(t)) B_{i} u(t) \\
& +\sum_{i=1}^{L} h_{i}(z(t)) B_{i} \mu(t) .
\end{aligned}
$$

Now we can use (11) to obtain

$$
\dot{x}(t)=A(t) x(t)+B(t) u(t)+B(t) \mu(t),
$$

where $A(t), B(t)$ and $C(t)$ are given in (11). This is exactly the dynamic behavior of the global system as described in (9), which shows that (9) does indeed describe the dynamic behavior of $x_{i}$. A similar method can be used to show that the premises of the lemma also result in

$$
y(t)=C(t) x(t) .
$$

\section{Estimation algorithm via a fuzzy robust observer}

3.1. Design of a T-S robust sliding mode observer. A robust Sliding Mode Observer (RSMO) is used to solve the state estimate problem for uncertain systems. In this section we modify the RSMO for the system given by (14) and (15), if the following assumption holds.

Assumption 1. The matrices $h_{i}(z(t)) B_{i}, C_{i}$ are full rank, $\left(A_{i}, C_{i}\right)$ are observable, uncertain vector functions are $h_{i}(z(t)) B_{i} \mu(t)=\Delta F_{i}$ and a scalar function $\alpha(t, y)$ is such that

$$
\bar{F}_{i}=\mu(t), \quad\left\|\Delta \bar{F}_{i}\right\| \leq r\|u(t)\|+\alpha\left(t, y_{i}\right),
$$

where $r$ is a known positive real coefficient.

There exists an exponentially convergent RSMO for the system described by (14) and (15), which is given by

$$
\begin{aligned}
\dot{\hat{x}}_{i} & =A_{i} \hat{x}_{i}+h_{i}(z(t)) B_{i} u-K_{i}\left(\hat{y}_{i}-y_{i}\right) \\
& +h_{i}(z(t)) B_{i} \nu, \\
\hat{y}_{i} & =C_{i} \hat{x}_{i},
\end{aligned}
$$


where $\dot{\hat{x}}_{i}$ is the $i$-th local state estimate $K_{i}$ is the observer feedback gain matrix, $\nu$ is the control input.

Let $e_{i}(t)=\hat{x}_{i}(t)-x_{i}(t)$. From (14), (15), (20) and (21), the dynamics of the observation error are given by

$$
\begin{aligned}
\frac{\mathrm{d}}{\mathrm{d} t} e_{i}= & \left(A_{i}-K_{i} C_{i}\right) e_{i}-h_{i}(z(t)) B_{i} \Delta \bar{F}_{i} \\
& +h_{i}(z(t)) B_{i} \nu
\end{aligned}
$$

Let $A_{i}^{0}=\left(A_{i}-K_{i} C_{i}\right)$. At a finite time, if state variables of the deviation system (22) are asymptotically convergent to the origin, then the states can be estimated by (14) and (15).

In order to design the RSMO which is given by (20), first, design the sliding mode surface as

$$
s_{i}=M_{i} e_{i}=F_{i} C_{i} e_{i}=F_{i}\left(C_{i} \hat{x}_{i}-y_{i}\right) .
$$

Hence, the parameter matrix $F_{i}$ is designed for the sliding mode surface.

In this paper, the following sliding mode strategy $\nu$ is used for RSMO (22):

$$
\nu= \begin{cases}0, & \text { if } \mid s_{i}^{T} M_{i} h_{i} B_{i} \|=0, \\ -\frac{\left(s_{i}^{T} M_{i} h_{i} B_{i}\right)^{T}}{\left\|s_{i}^{T} M_{i} h_{i} B_{i}\right\|}\left(\rho_{i}\left\|s_{i}\right\|\left\|M_{i} h_{i} B_{i}\right\|+\Delta_{i}\right), & \text { otherwise, }\end{cases}
$$

where

$$
\Delta_{i}=\eta\left(\frac{1}{2}\right)^{\beta}\left\|s_{i}\right\|^{2 \beta}
$$

and the parameters $\beta>0,0<\eta<1, \rho_{i}=r\|u(t)\|+$ $\alpha\left(t, y_{i}\right)$.

In order to facilitate the proof, let $e_{i}=\left[e_{i}^{1}, e_{i}^{2}\right]^{T}$. The error system (22) can be written as follows:

$$
\begin{aligned}
\dot{e}_{i}^{1}(t)= & A_{i}^{011} e_{i}^{1}(t)+A_{i}^{012} e_{i}^{2}(t), \\
\dot{e}_{i}^{2}(t)= & A_{i}^{021} e_{i}^{1}(t)+A_{i}^{022} e_{i}^{2}(t) \\
& -h_{i} B_{i 2} \bar{F}_{i}+h_{i} B_{i 2} \nu,
\end{aligned}
$$

where $\left[A_{011}, A_{012} ; A_{021}, A_{022}\right]=A_{0},\left[0, B_{i 2}\right]^{T}=B_{i}$. The sliding mode (22) can be written as follows:

$$
s_{i}=M_{i 1} e_{i}^{1}+M_{i 2} e_{i}^{2},
$$

where $\left[M_{i 1}, M_{i 2}\right]=M_{i}$. Define the following matrices:

$$
\begin{aligned}
A_{i}^{M} & =A_{i}^{011}-A_{i}^{012} M_{i 2}^{-1} M_{i 1}, \\
A_{i}^{s} & =\frac{1}{2}\left(M_{i}^{T} M_{i} A_{i}^{0}+A_{i}^{0 T} M_{i}^{T} M_{i}\right) .
\end{aligned}
$$

The following theorem provides the design method of the robust sliding-mode observer (20). The designed observer is robust with respect to nonlinear uncertain parts, and can asymptotically estimate the state of the system (14).
Theorem 1. For an RSMO, using the sliding mode (23) and the control input of the observer (24) to design the parameter matrix $K_{i}$ and sliding mode parameter matrix $M_{i}$, respectively, make $A_{i}^{0}$ and $A_{i}^{M}$ Hurwitz matrices. Moreover, $\lambda_{\max }\left(A_{i}^{s}\right) \leq 0$, where $\lambda_{\max }(\cdot)$ denotes the maximum eigenvalue of matrix. Then RSMO is robust to nonlinear uncertain parts $\Delta F_{i}$, and the states of the system (14) can be estimated asymptotically. The convergence speed of the error system (22) is determined by the eigenvalues of $A_{i}^{M}$.

Proof. Consider the following Lyapunov function:

$$
V(t)=\frac{1}{2} s_{i}^{T} s_{i}=\frac{1}{2} e_{i}^{T} M_{i}^{T} M_{i} e_{i}
$$

Its time derivative with respect to (30) is

$$
\begin{aligned}
\dot{V}(t)= & s_{i}^{T} \dot{s}_{i}=e_{i}^{T} M_{i}^{T} M_{i} e_{i} \\
= & e_{i}^{T} M_{i}^{T} M_{i}\left(A_{i}^{0} e_{i}-h_{i} B_{i} \Delta \bar{F}_{i}+h_{i} B_{i} \nu\right) \\
= & \frac{1}{2} e_{i}^{T}\left(M_{i}^{T} M_{i} A_{i}^{0}+A_{i}^{0} M_{i}^{T} M_{i}\right) e_{i} \\
& -s_{i}^{T} M_{i} h_{i} B_{i} \Delta \bar{F}_{i}+s_{i}^{T} M_{i} h_{i} B_{i} \nu \\
\leq & \lambda_{\max }\left(A_{i}^{s}\right)\left\|e_{i}\right\|^{2}-s_{i}^{T} M_{i} h_{i} B_{i} \Delta \bar{F}_{i}+s_{i}^{T} M_{i} h_{i} B_{i} \nu \\
\leq & \left\|s_{i}\right\|\left\|M_{i} h_{i} B_{i}\right\|\left\|\Delta \bar{F}_{i}\right\| \\
& -\left(\rho_{i}\left\|s_{i}\right\|\left\|M_{i} h_{i} B_{i}\right\|+\eta\left(\frac{1}{2}\right)^{\beta}\left\|s_{i}\right\|^{2 \beta}\right) .
\end{aligned}
$$

By (19) and (24), design the parameter $\rho_{i} \geq\left\|\Delta \bar{F}_{i}\right\|$. Hence

$$
\begin{aligned}
\dot{V}(t) & \leq-\left(\frac{1}{2}\right)^{\beta} \eta\left\|s_{i}\right\|^{2 \beta} \leq-\eta V^{\beta}, \quad \forall t \geq 0 \\
V(0) & \geq 0
\end{aligned}
$$

The error system (22) can be obtained on sliding the surface $s_{i}=0$ in a limit time $T_{r}, T_{r}=[\eta(1-\beta)]^{-1} V^{1-\beta}(0)$. When the system (34) reaches the sliding surface $s_{i}=0$, the dynamic performance of the system (22) is decided by the linear sliding mode (23). Through Eqn. (27), we can obtain $s_{i}=M_{i 1} e_{i}^{1}+M_{i 2} e_{i}^{2}=0$, or $e_{i}^{2}=M_{i 2}^{-1} M_{i 1} e_{i}^{1}$, substituting it into (33), and obtain the reduced order system equation

$$
\dot{e}_{i}^{1}(t)=\left(A_{i}^{011}-A_{i}^{012} M_{i 2}^{-1} M_{i 1}\right) e_{i}^{1}(t)=A_{i}^{M} e_{i}^{1}(t) .
$$

The design parameters of the sliding matrix $M_{i}$ make $A_{i}^{M}$ a Hurwitz matrix, so that the system (33) is asymptotically stable. The convergence speed of the error system (22) is determined by the eigenvalues of $A_{i}^{M}$. Hence, the observer is robust to nonlinear uncertain parts and can be used to estimate the state of the system (14).

We combine the state observer which is described by Eqns. (20) and (21) for the local systems given in (14) and (15) to obtain a state estimator for the T-S fuzzy model given in (3) and (4). Since we know from (13) that 
$x(t)=\sum_{i=1}^{L} x_{i}(t)$, we can combine the local state estimates in (20) to estimate the state of the T-S fuzzy model (3) and (4)

$$
\hat{x}(t)=\sum_{i=1}^{L} \hat{x}_{i}(t)
$$

Theorem 2. The state estimation given by (20) and (34) is an unbiased estimate of the true state of the T-S fuzzy model given by (3) and (4).

Proof. We can use (13) and (34) to derive the error in the state estimate as

$$
\tilde{x}(t)=x(t)-\hat{x}(t)=\sum_{i=1}^{L} x_{i}(t)-\sum_{i=1}^{L} \hat{x}_{i}(t) .
$$

Therefore, knowing from Theorem 1 that $\lim _{t \rightarrow \infty}\left(x_{i}(t)-\right.$ $\left.\hat{x}_{i}(t)\right)=0$, we obtain

$$
\lim _{t \rightarrow \infty} \tilde{x}(t)=0 .
$$

Remark 1. An important advantage of a T-S fuzzy system is its universal approximation of any smooth nonlinear function by blending some local linear system models. This greatly facilitates the analysis and synthesis of the complex nonlinear system. Considering the advantage of the T-S fuzzy system, in this paper, a robust sliding mode observer based on a T-S fuzzy system is to be designed for nonlinear systems.

3.2. Determination of the inverse system. Consider the nonlinear system (2). It is defined as the system $S_{0}$, and it is described by the following equation:

$$
S_{0}:\left\{\begin{array}{c}
\dot{x}=f(x, u+\mu), \\
g_{0}(y, x)=0,
\end{array}\right.
$$

where $g_{0}(y, x)=y-h(x)$, and $x \in M_{0} \subset \mathbb{R}^{r}, u \in$ $L_{0} \subset \mathbb{R}^{m}, y \in N_{0} \subset \mathbb{R}^{n}$. Then, a system sequence $S_{1}$, $S_{2}, \ldots, S_{k}, \ldots$ is defined in the recursive way from $S_{0}$. Generally, $S_{k}$ can be described by

$$
S_{k}:\left\{\begin{array}{l}
\dot{x}=f(x, u+\mu), \\
g_{k}\left(y, y^{\prime}, \ldots, y^{(k)}, x, u+\mu\right)=0,
\end{array}\right.
$$

where $x \in M_{k}, u \in L_{k},\left[y, y^{\prime}, \ldots, y^{(k)}\right] \in N_{k}$.

Set

$\eta_{k}=\max _{\Omega_{k}}\left\{\operatorname{rank}\left[\frac{\partial}{\partial(u+\mu)} g_{k}\left(y, y^{\prime}, \ldots, y^{k}, x, u+\mu\right)\right]\right\}$,

where $\Omega_{k}=\left[L_{k}, M_{k}, N_{k}\right]$,

$$
M_{k+1}=\left\{x \mid x \in M_{k}, \operatorname{rank}\left[\frac{\partial}{\partial(u+\mu)} h_{k}(\cdot)\right]=\eta_{k}\right\} \text {, }
$$

$$
\begin{gathered}
L_{k+1}=\left\{u+\mu \mid u+\mu \in L_{k},\right. \\
\left.\operatorname{rank}\left[\frac{\partial}{\partial(u+\mu)} \quad h_{k}(\cdot)\right]=\eta_{k}\right\}, \\
\bar{N}_{k+1}=\left\{y, \ldots, y^{(k)} \mid\left(y, \ldots, y^{(k)}\right) \in N_{k},\right. \\
\left.\operatorname{rank}\left[\frac{\partial}{\partial(u+\mu)} h_{k}(\cdot)\right]=\eta_{k}\right\} .
\end{gathered}
$$

From (38), it follows that the disturbance/fault is given by

$$
u+\mu=g_{k}^{-1}\left(x, y, y^{\prime}, \ldots, y^{k}\right)=u_{c},
$$

and $u_{c}$ can be obtained by the above equation. However, the actual state must be observed while the state is unmeasurable. Consequently $\hat{u}_{c} \rightarrow u_{c}$ as $\hat{x} \rightarrow x$ by the following theorem.

Theorem 3. Let the observer

$$
\hat{u}_{c}=g_{k}^{-1}\left(\hat{x}, y, y^{\prime}, \ldots, y^{(k)}\right)
$$

be used to estimate the input vector $u_{c}$. If the state estimate of $\hat{x} \rightarrow x$, then $\hat{u}_{c} \rightarrow u_{c}$.

Proof. Let $e=\hat{x}-x$. Then the observer $u_{c}$ can be described as

$$
\begin{aligned}
\hat{u}_{c} & =g_{k}^{-1}\left(x+e, y, y^{\prime}, \ldots, y^{(k)}\right) \\
& =g_{k}^{-1}\left(x, y, y^{\prime}, \ldots, y^{(k)}, e\right)
\end{aligned}
$$

Obviously, the function $g_{k}^{-1}(\cdot)$ has the properties

$$
\left.g_{k}^{-1}\left(x, y, y^{\prime}, \ldots, y^{(k)}, e\right)\right|_{e=0}=g_{k}^{-1}\left(x, y, y^{\prime}, \ldots, y^{k}\right) \text {. }
$$

Hence, the function $g_{k}^{-1}\left(x, y, y^{\prime}, \cdots, y^{(k)}, e\right)$ can be expanded in a Taylor polynomial at $e=0$. Thus

$$
\hat{u}_{c}=\left.g_{k}^{-1}\left(x, y, y^{\prime}, \cdots, y^{k}, e\right)\right|_{e=0}+\frac{\partial\left(g_{k}^{-1}\right)}{\partial x} e+o\left(e^{2}\right)
$$

where

$$
\frac{\partial\left(g_{k}^{-1}\right)}{\partial x} \triangleq \frac{\partial\left(g_{k}^{-1}\left(x, y, y^{\prime}, \ldots, y^{(k)}\right)\right)}{\partial x} .
$$

If $\partial\left(g_{k}^{-1}\right) / \partial x$ is bounded, namely,

$$
\left|\frac{\partial\left(g_{k}^{-1}\right)}{\partial x}\right| \leq \sigma,
$$

it is easy to see that if $\hat{x} \rightarrow x$, then $\hat{u}_{c} \rightarrow u_{c}$.

Remark 2. In the actual plant, $\left|\partial\left(g_{k}^{-1}\right) / \partial x\right|$ is bounded, and

$$
\lim _{e \rightarrow 0} \frac{\partial\left(g_{k}^{-1}\right) / \partial x}{e}=0 .
$$


3.3. Actuator fault estimation - Because of the design of an observer for uncertain systems, the actual state can be estimated by an RSMO when a fault occurs. In this section, we propose a novel fault detection and estimation strategy based on an inverse system method. From (40), it follows that the actual fault is given by

$$
\mu=g_{k}^{-1}\left(x, y, y^{\prime}, \ldots, y^{k}\right)-u(t) .
$$

It is easy to see that the state, output and high-order derivative of the output are the input in (44). In real-world systems, the $k$-th derivative of the output $y$ is unavailable, so we introduce the following:

$$
\begin{aligned}
{\left[\begin{array}{c}
\dot{y}_{e} \\
\ddot{y}_{e} \\
y_{e}^{(3)} \\
\vdots \\
y_{e}^{(a)}
\end{array}\right]=} & {\left[\begin{array}{ccccc}
-\frac{1}{\tau} & 0 & 0 & \cdots & 0 \\
-\frac{1}{\tau^{2}} & -\frac{1}{\tau} & 0 & \ddots & 0 \\
-\frac{1}{\tau^{3}} & -\frac{1}{\tau^{2}} & -\frac{1}{\tau} & \ddots & 0 \\
\vdots & \vdots & \vdots & \ddots & \vdots \\
-\frac{1}{\tau^{a-1}}-\frac{1}{\tau^{a-2}} & -\frac{1}{\tau^{a-3}} & \ldots & -\frac{1}{\tau}
\end{array}\right] } \\
& \times\left[\begin{array}{c}
y_{e} \\
\dot{y}_{e} \\
\ddot{y}_{e} \\
\vdots \\
\frac{1}{\tau} \\
y_{e}^{(a-1)}
\end{array}\right]+\left[\begin{array}{c}
\frac{1}{\tau^{2}} \\
\frac{1}{\tau^{3}} \\
\vdots \\
\frac{1}{\tau^{a-1}}
\end{array}\right] y .
\end{aligned}
$$

Note that (45) is obtained by repeated use of the filter $\tau \dot{\omega}+\omega=\nu$ to obtain $\dot{\omega}$ as a filtered derivative of $\nu$. The successive derivatives of the output $y$ shown in Eqn. (45) are obtained by the repeated use of such a filter. Hence, as $\tau \rightarrow 0, y_{e}^{(k)} \rightarrow y^{(k)}, k=1, \ldots, a$. Thus the following equation can be obtained for the actuator fault:

$$
\hat{\mu}=g_{k}^{-1}\left(\hat{x}, y, y_{e}^{\prime}, \ldots, y_{e}^{k}\right)-u(t)
$$

Therefore, according to Theorem 2 and (46), where $\hat{x} \rightarrow$ $x$ and $y_{e}^{(k)} \rightarrow y^{(k)}$, we obtain $\hat{\mu} \rightarrow \mu$.

A flow chart in Fig. 1 gives a clear idea of the overall design procedure,

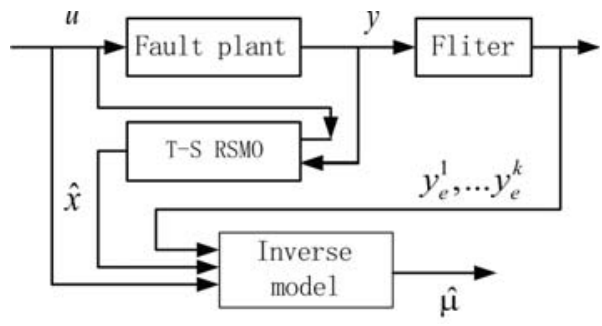

Fig. 1. Actuator fault estimate scheme via a T-S robust sliding mode observer.

Remark 3. LED is used to design the estimation algorithm for the system for an actuator fault. Compared with the results of Yan and Edwards (2007), the estimation algorithm does not need to know the bounds of the disturbance. The estimation algorithm can be applied to FTC for a nonlinear uncertain system, while the information of the estimate is used to accommodate the control command.

\section{Estimation algorithm based on an integrated fuzzy observer and an inverse system}

Fault estimation based on an adaptive observer has good accuracy and robustness for unknown parameters. At the same time, fault estimation based on an adaptive observer is based ona pure integral term. However, the speed and accuracy of fault estimation cannot be satisfactory. For some of the above problems, Zhang et al. (2009) proposed a fast adaptive fault estimation. However, this method is mainly focused on linear time invariant systems. Indeed, the established form of the actuator fault may be very time-varying, so estimation based on an adaptive observer may not be satisfactory to estimate rapid time-varying parameters. Sliding mode observer-based fault estimation has a proportional term at heart, which can quickly ensure the requirement for a time-varying fault. However, a steady-state error will exist for a constant fault.

In this section, the second observer algorithm is proposed based on a combined fuzzy observer and an inverse system without adaptive and sliding mode techniques.

4.1. Inverse system of the T-S fuzzy form. The fuzzy inverse model or the T-S inverse model is used to control nonlinear systems (e.g., Babuska, 1998; Boukezzoula et al., 2003; 2007), mainly for input and output data identification. Essentially, fuzzy inverse control is a data-driven control method primarily for SingleInput Single-Output (SISO) system. For Multiple-Input Multiple-Output (MIMO) systems, in this section a form of a T-S fuzzy inverse system is given by a dynamic inverse method to design $K(t)=\sum_{i=1}^{L} K_{i} h_{i}$.

Consider the system in (17). Let the vector relative degree of the system from the output $y \in \mathbb{R}^{n}$ to the input $u \in \mathbb{R}^{m}$ be $\left[\begin{array}{ll}r_{u 1} & r_{u 2}, \ldots, r_{u n}\end{array}\right]$. Hence

$$
\begin{aligned}
C^{j} A^{k} B & =0, \quad k=0,1, \ldots, r_{u j}-2, \\
C^{j} A^{r_{u j}-1} B & \neq 0, \quad j=0,1, \ldots, n .
\end{aligned}
$$

It follows that

$$
\begin{gathered}
y_{j}^{r_{u j}}=\left(C^{j} A^{r_{u j}}\right) x(t)+\left(C^{j} A^{r_{u j}-1} B\right)(u(t)+\mu(t)), \\
j=0,1, \ldots, n .
\end{gathered}
$$


Hence

$$
\begin{aligned}
{\left[\begin{array}{c}
y_{1}^{r_{u 1}} \\
y_{2}^{r_{u 2}} \\
\vdots \\
y_{m}^{r_{u m}}
\end{array}\right]=} & {\left[\begin{array}{c}
C^{1} A^{r_{u 1}-1} B \\
C^{2} A^{r_{u 2}-1} B \\
\vdots \\
C^{m} A^{r_{u m}-1} B
\end{array}\right](u(t)+\mu(t)) } \\
& +\left[\begin{array}{c}
C^{1} A^{r_{u 1}} x \\
C^{2} A^{r_{u 2}} x \\
\vdots \\
C^{m} A^{r_{u m}} x
\end{array}\right]
\end{aligned}
$$

where $y_{j}$ is the $j$-th output of $y$. From (50), it follows that the actuator fault is given by

$$
\begin{aligned}
\mu(t)= & {\left[\begin{array}{c}
C^{1} A^{r_{u 1}-1} B \\
C^{2} A^{r_{u 2}-1} B \\
\vdots \\
C^{m} A^{r_{u m}-1} B
\end{array}\right] } \\
& \times\left\{\left[\begin{array}{c}
y_{1}^{r_{u 1}} \\
y_{2}^{r_{u 2}} \\
\vdots \\
y_{m}^{r_{u m}}
\end{array}\right]-\left[\begin{array}{c}
C^{1} A^{r_{u 1}} x \\
C^{2} A^{r_{u 2}} x \\
\vdots \\
C^{m} A^{r_{u m}} x
\end{array}\right]\right\}-u(t) .
\end{aligned}
$$

\subsection{Estimation algorithm via an inverse-based fuzzy} observer. In this section, an estimation algorithm via an inverse-based fuzzy observer is proposed in the following theorem. The same effect can be achieved as in the first estimation algorithm. However, no adaptive or sliding mode technologies are used in the design.

Theorem 4. Consider the following observer and actuator fault estimator:

$$
\begin{aligned}
\dot{\hat{x}}(t)= & \sum_{i=1}^{L} h_{i}(z)\left[A_{i} \hat{x}(t)+B_{i} u(t)\right. \\
& \left.-K_{i}(\hat{y}(t)-y(t))+B_{i} \hat{\mu}(t)\right], \\
\hat{\mu}(t)= & {\left[\begin{array}{c}
C^{1} A^{r_{u 1}-1} B \\
C^{2} A^{r_{u 2}-1} B \\
\vdots \\
C^{m} A^{r_{u m}-1} B
\end{array}\right]-1 } \\
& \times\left\{\left[\begin{array}{c}
y_{e 1}^{r_{u 1}} \\
y_{e 2}^{r_{u 2}} \\
\vdots \\
y_{e m}^{r_{u m}}
\end{array}\right]-\left[\begin{array}{c}
C^{1} A^{r_{u 1}} \hat{x} \\
C^{2} A^{r_{u 2}} \hat{x} \\
\vdots \\
C^{m} A^{r_{u m}} \hat{x}
\end{array}\right]\right\}-u(t) .
\end{aligned}
$$

Suppose it is used to estimate the state vector $\hat{x}$ and the magnitude of the actuator fault $\hat{\mu}$. From the filter equation (45), let the observer gain matrix $K_{i}$ be chosen such that the system

$$
\begin{aligned}
\dot{\tilde{x}}= & \sum_{i=1}^{L} \sum_{j=1}^{L} h_{i} h_{j}\left(A_{i}-K_{i} C_{j}-B_{i}\right. \\
& \left.\times\left[\begin{array}{c}
C_{1} A^{r_{u 1}-1} B \\
\vdots \\
C_{m} A^{r_{u m}-1} B
\end{array}\right]^{-1}\left[\begin{array}{c}
C^{1} A^{r_{u 1}} \\
\vdots \\
C^{m} A^{r_{u m}}
\end{array}\right]\right) \tilde{x}
\end{aligned}
$$

is stable. Then the filter constant $\tau \rightarrow 0, \hat{\mu} \rightarrow \mu$ and $\hat{x} \rightarrow x$ as $t \rightarrow \infty$.

Proof. Let $\tilde{x}=\hat{x}-x$ be the estimation error, while (51) and (53) are substituted into the equation for the estimation error. Then

$$
\begin{aligned}
\dot{\tilde{x}}= & \sum_{i=1}^{L} \sum_{j=1}^{L} h_{i} h_{j}\left[\left(A_{i}-K_{i} C_{j}\right) \tilde{x}+B_{i}(\hat{\mu}-\mu)\right] \\
= & \sum_{i=1}^{L} \sum_{j=1}^{L} h_{i} h_{j}\left(A_{i}-K_{i} C_{j}-B_{i}\right. \\
& \left.\times\left[\begin{array}{c}
C_{1} A^{r_{u 1}-1} B \\
\vdots \\
C_{m} A^{r_{u m}-1} B
\end{array}\right]^{-1}\left[\begin{array}{c}
C^{1} A^{r_{u 1}} \\
\vdots \\
C^{m} A^{r_{u m}}
\end{array}\right]\right) \tilde{x} \\
& +\left[\begin{array}{c}
C_{1} A^{r_{u 1}-1} B \\
\vdots \\
C_{m} A^{r_{u m}-1} B
\end{array}\right]^{-1}\left[\begin{array}{c}
y_{e 1}^{r_{u 1}}-y_{1}^{r_{u 1}} \\
\vdots \\
y_{e m}^{r_{u m}}-y_{m}^{r_{u m}}
\end{array}\right] .
\end{aligned}
$$

Hence, as $\tau \rightarrow 0$, we get $y_{e}^{(r)} \rightarrow y^{(r)}$. Thus it follows that, as $t \rightarrow \infty, \hat{\mu} \rightarrow \mu$ and $\hat{x} \rightarrow x$.

The estimation error equation (54) can be represented as

$$
\dot{\tilde{x}}=\sum_{i=1}^{L} \sum_{j=1}^{L} h_{i} h_{j}\left(\bar{A}_{i}-K_{i} C_{j}\right) \tilde{x},
$$

where

$$
\bar{A}_{i}=A_{i}-B_{i}\left[\begin{array}{c}
C_{1} A^{r_{u 1}-1} B \\
\vdots \\
C_{m} A^{r_{u m}-1} B
\end{array}\right]^{-1}\left[\begin{array}{c}
C^{1} A^{r_{u 1}} \\
\vdots \\
C^{m} A^{r_{u m}}
\end{array}\right] .
$$

To design the parameters matrix $K_{i}$, make $\bar{A}_{i}-K_{i} C_{i}$ a Hurwitz matrix. The matrix $K_{i}$ can guarantee the asymptotical stability of the estimation error (55) via a T-S fuzzy model in the case of actuator faults. At same time, the actuator fault value can be estimated.

To give a clear idea of the overall design procedure, we provide a flow chart in Fig. 2.

Remark 4. An important advantage of a T-S fuzzy system is its universal approximation of any smooth nonlinear function by "blending" some local linear system models. This greatly facilitates the analysis and synthesis of 


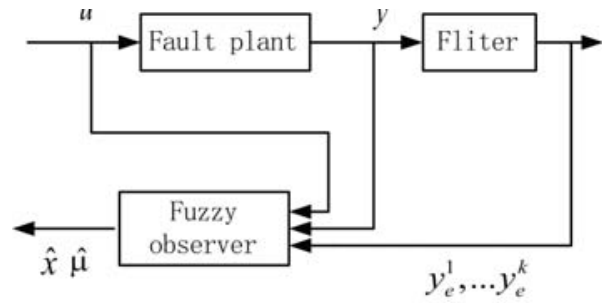

Fig. 2. Disturbance/fault estimate scheme via an integrated T-S fuzzy observer and inverse system.

complex nonlinear systems. Consequently, Theorem 3 in Section 4 is only used to design the observer time-varying feedback gain $K(t)=\sum_{i=1}^{L} h_{i}(z) K_{i}$. Actually, Eqns. (52) and (53) are replaced as follows:

$$
\begin{aligned}
\dot{\hat{x}}(t) & =f(\hat{x},(u+\hat{\mu}))-\sum_{i=1}^{L} h_{i}(z)\left[K_{i}(\hat{y}-y)\right], \\
\hat{\mu} & =g_{k}^{-1}\left(\hat{x}, y, y_{e}^{\prime}, \ldots, y_{e}^{k}\right)-u(t) .
\end{aligned}
$$

Remark 5. The estimation algorithm which is presented in this section does not require the system to be minimum phase, which not only provides information for fault detection but also enables estimation of an actuator fault. Compared with the first estimation method in Section 3, it does not need adaptive and sliding mode techniques, and parameter selection is easy, i.e., only the parameter matrix $K_{i}$ have to be designed to make $\bar{A}_{i}-K_{i} C_{i}$ a Hurwitz matrix. Compared with the results of Yan and Edwards (2007) as well as Lendek et al. (2010a), this estimation method is much simpler in design and easier to realize.

Remark 6. Geometric theory is used to design the fault observer based on decoupling techniques in the works of Kabore and Wang (2001) as well as Kabore et al. (2000). This will make the design process complicated. Hence these methods are difficult to promote for engineering applications. Some differences between the approach of Kabore and Wang (2001) and Kabore et al. (2000) and ours concern two aspects:

- The fault diagnosis approaches of Kabore and Wang (2001) as well as Kabore et al. (2000) were presented for affine systems based on decoupling techniques, but our study is focused on general nonlinear systems and uses the T-S fuzzy approach.

- Our paper deals with actuator failures, while the above scientists studied system faults in their works. Hence, the results presented in our paper have wider application potentials, which cover more general practical systems.

\section{Illustrative examples}

5.1. Example 1. In this section, the first proposed estimation strategy will be demonstrated with an example, which is a nonlinear continuous system taken from the work of Chang and Yeh (2006). It is described as follows under the actuator fault:

$$
\begin{aligned}
\dot{x}_{1}(t)= & \left\{-21.96+21.96 \cdot \cos \left(x_{1}(t)\right)\right\} \cdot \sin \left(x_{1}(t)\right) \\
& +6.1-5.55 \cos \left(x_{1}(t)\right) \cdot x_{2}(t)+\omega_{1}(t) \\
& +\frac{u(t)+\mu(t)}{1.54-0.54 \cdot \cos \left(x_{1}(t)\right)}, \\
\dot{x}_{2}(t)= & 3 x_{1}(t)+0.11 x_{2}(t)+\omega_{2}(t), \\
y(t)= & 3 x_{1}(t)+2 x_{2}(t) .
\end{aligned}
$$

We apply the technique called local approximation in fuzzy partition space and presented by Tanaka and Wang (2001) to construct the structure of a T-S fuzzy model. The spirit of this method is to approximate a nonlinear term by judiciously chosen linear terms. In this example, we assume that the state variable $x_{1}(t)$ is measurable and its range is of $x_{1}(t) \in[-\pi / 2, \pi / 2]$. Besides, the nonlinear terms $\sin \left(x_{1}(t)\right)$ and $\cos \left(x_{1}(t)\right)$ in nonlinear system equations (57) are approximated by the following two rules:

Rule 1: When $x_{1}(t) \rightarrow 0$, it is assumed that $\sin \left(x_{1}(t)\right) \rightarrow$ $x_{1}(t)$ and $\cos \left(x_{1}(t)\right) \rightarrow 1$.

Rule 2: When $x_{1}(t) \rightarrow \pm \pi / 2\left(\left|x_{1}(t)\right|<\pi / 2\right)$, it is assumed that $\sin \left(x_{1}(t)\right) \rightarrow 2 / \pi x_{1}(t)$ and $\cos \left(x_{1}(t)\right) \rightarrow$ $\cos \left(88^{\circ} \times \pi / 180^{\circ}\right)$.

Based on the above representation, one can obtain the following two-rule (i.e., $L=2$ ) T-S fuzzy model.

Plant Rule 1: IF $x_{1}(t)$ is about 0 , THEN

$$
\begin{aligned}
& \dot{x}(t)=A_{1} x(t)+B_{1} u(t)+B_{1} \mu(t), \\
& y(t)=C_{1} x(t) .
\end{aligned}
$$

Plant Rule 2: IF $x_{1}(t)$ is about $\pm \pi / 2\left(\left|x_{1}(t)\right|<\pi / 2\right)$, THEN

$$
\begin{aligned}
& \dot{x}(t)=A_{2} x(t)+B_{2} u(t)+B_{2} \mu(t) \\
& y(t)=C_{2} x(t)
\end{aligned}
$$

where

$$
\begin{array}{lll}
A_{1}=\left(\begin{array}{ll}
0 & 0.55 \\
3 & 0.11
\end{array}\right), & A_{2}=\left(\begin{array}{cc}
-13.98 & 5.9063 \\
3 & 0.11
\end{array}\right), \\
B_{1}=\left(\begin{array}{l}
1 \\
0
\end{array}\right), & B_{2}=\left(\begin{array}{c}
0.65 \\
0
\end{array}\right) .
\end{array}
$$

We can choose the control input as

$$
u(t)=\sum_{i=1}^{L} h_{i}(z) \mathcal{K}_{i} \hat{x}(t) .
$$

Let us use the feedback control gains $\mathcal{K}_{i}$ of Chang and Yeh (2006),

$$
\begin{aligned}
& \mathcal{K}_{1}=\left(\begin{array}{ll}
-3.8753 & -5.2832
\end{array}\right) \\
& \mathcal{K}_{2}=\left(\begin{array}{ll}
12.1769 & -11.7884
\end{array}\right)
\end{aligned}
$$



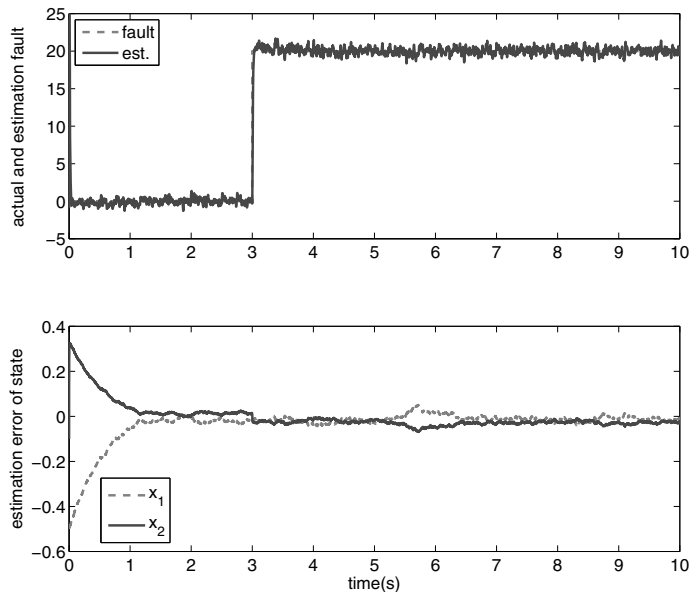

Fig. 3. Unknown constant fault estimate and the observer state error.

By solving the derived stability conditions of Theorem 1, the designed observer gains are

$$
\begin{aligned}
& K_{1}=\left(\begin{array}{ll}
0.340 & 0.0854
\end{array}\right)^{T}, \\
& K_{2}=\left(\begin{array}{ll}
0.340 & 0.0854
\end{array}\right)^{T} .
\end{aligned}
$$

The parameter matrices $F$ of the sliding mode surface which is defined by (35) can be obtained as $F_{1}=$ 3.31 and $F_{2}=4.46$. The parameters of the sliding mode surface are selected as $\beta=10, \eta=0.1, \rho_{i}=5$ by Eqn. (36), and the filter parameters as $\tau=0.005$. It is supposed that the unknown disturbance $\omega(t)$ is band-limited white noise with power 0.001 . Using the method proposed in Section 3, we can calculate the inverse system of (57). We apply the above observed-state feedback control gains $\mathcal{K}_{1}$ and $\mathcal{K}_{2}$, the observer gains $K_{1}$ and $K_{2}$, and a constant actuator fault is assumed as

$$
\mu(t)=\left\{\begin{array}{cl}
0, & 0 \leq t<3 \\
20, & 3 \leq t \leq 10
\end{array}\right.
$$

As seen in Fig. 3, the actuator fault and the states are estimated well with the first estimation approach. In simulation, the initial states are given as $\left[x_{1}(0), x_{2}(0)\right]^{T}=$ $[0.1,0.5]^{T}$.

Secondly, it is assumed that a time-varying actuator fault $\mu(t)$ appears as

$$
\mu(t)= \begin{cases}0, & 0 \leq t<3 \\ 20 \sin (\pi t), & 3 \leq t \leq 10 .\end{cases}
$$

The estimation of a time-varying actuator fault and the error of states are displayed in Fig. 4.

5.2. Example 2. In this subsection, we test the proposed second estimation strategy of a two-link planar
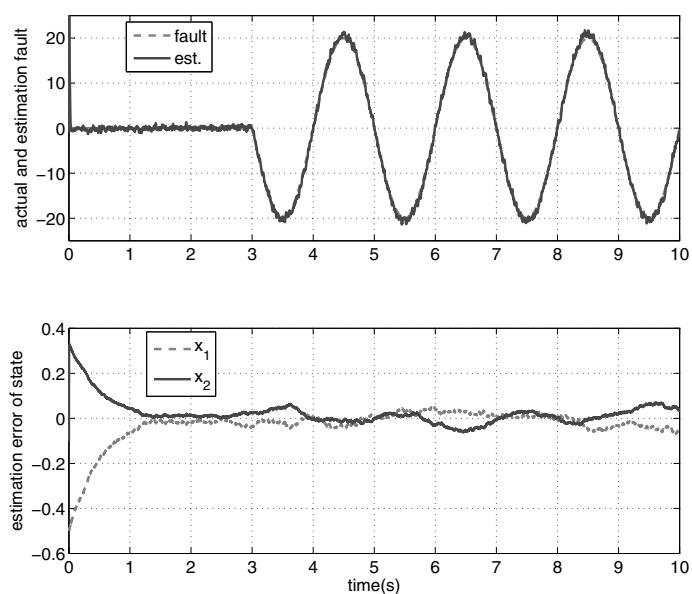

Fig. 4. Unknown time-varying fault estimate and the observer state error.

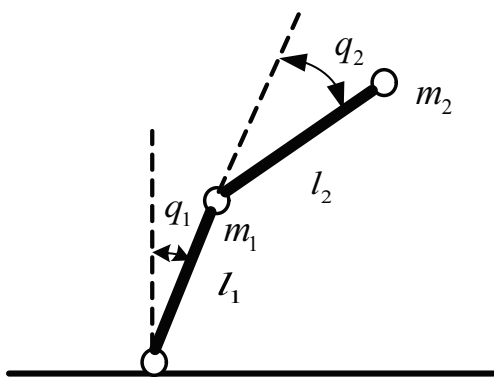

Fig. 5. Configuration of two-link robot systems.

robot manipulator with an actuator fault, cf. Fig. 5. The dynamic equation of the two-link robot system is given as follows:

$$
M(q) \ddot{q}+C(q, \dot{q}) \dot{q}+G(q)=\tau,
$$

where

$$
\begin{gathered}
M(q)=\left[\begin{array}{cc}
\left(m_{1}+m_{2}\right) l_{1}^{2} & m_{2} l_{1} l_{2}\left(s_{1} s_{2}+c_{1} c_{2}\right) \\
m_{2} l_{1} l_{2}\left(s_{1} s_{2}+c_{1} c_{2}\right) & m_{2} l_{2}^{2}
\end{array}\right], \\
C(q, \dot{q})=m_{2} l_{1} l_{2}\left(c_{1} s_{2}-s_{1} c_{2}\right)\left[\begin{array}{cc}
0 & \dot{q}_{2} \\
-\dot{q}_{1} & 0
\end{array}\right], \\
G(q)=\left[\begin{array}{cc}
-\left(m_{1}+m_{2}\right) l_{1} g s_{1} \\
-m_{2} l_{2} g s_{2} & 0
\end{array}\right],
\end{gathered}
$$

$q=\left[q_{1}, q_{2}\right], q_{1}$ and $q_{2}$ are generalized coordinates, $M(q)$ is the moment of inertia, $C(q, \dot{q})$ includes Coriolis and centripetal forces, and $G(q)$ is the gravitational force. Other quantities include link masses $m_{1}, m_{2}$, link lengths $l_{1}, l_{2}$, angular positions $q_{1}, q_{2}$, torques applied $\tau=$ $\left[\tau_{1}, \tau_{2}\right]^{T}$, acceleration due to gravity $g=9.8\left(\mathrm{~m} / \mathrm{s}^{2}\right)$. For brevity, we use the notation $s_{1}=\sin \left(q_{1}\right), s_{2}=\sin \left(q_{2}\right)$, $c_{1}=\cos \left(q_{1}\right)$ and $c_{2}=\cos \left(q_{2}\right)$. Let $x_{1}=q_{1}, x_{2}=q_{2}$, $x_{3}=\dot{q}_{1}$ and $x_{4}=\dot{q}_{2}$. 
For all simulations, the parameters are given in Table 1 . The initial conditions are assumed to be $x(0)=$

Table 1. Parameters of the two link-robot.

\begin{tabular}{|c|l|l|}
\hline Inertia parameter & Link 1 & Link 2 \\
\hline \hline$m(\mathrm{~kg})$ & 1.0 & 1.0 \\
\hline$l(\mathrm{~m})$ & 1.0 & 1.0 \\
\hline
\end{tabular}

$[1,1,0,0]^{T}$ and $\hat{x}(0)=[0,0,0,0]^{T}$.

The T-S fuzzy model for the system in (58) is given by the following nine-rule fuzzy model:

Rule 1: IF $x_{1}(t)$ is about $-\pi / 2$ and $x_{2}$ is about $-\pi / 2$, THEN

$$
\dot{x}(t)=A_{1} x(t)+B_{1} u(t)+\omega(t), \quad y(t)=C_{1} x(t) .
$$

Rule 2: IF $x_{1}(t)$ is about $-\pi / 2$ and $x_{2}$ is about 0 , THEN

$$
\dot{x}(t)=A_{2} x(t)+B_{2} u(t)+\omega(t), \quad y(t)=C_{2} x(t) .
$$

Rule 3: IF $x_{1}(t)$ is about $-\pi / 2$ and $x_{2}$ is about $\pi / 2$, THEN

$$
\dot{x}(t)=A_{3} x(t)+B_{3} u(t)+\omega(t), \quad y(t)=C_{3} x(t) .
$$

Rule 4: IF $x_{1}(t)$ is about 0 and $x_{2}$ is about $-\pi / 2$, THEN

$$
\dot{x}(t)=A_{4} x(t)+B_{4} u(t)+\omega(t), \quad y(t)=C_{4} x(t) .
$$

Rule 5: IF $x_{1}(t)$ is about 0 and $x_{2}$ is about 0 , THEN

$$
\dot{x}(t)=A_{5} x(t)+B_{5} u(t)+\omega(t), \quad y(t)=C_{5} x(t) .
$$

Rule 6: IF $x_{1}(t)$ is about 0 and $x_{2}$ is about $\pi / 2$, THEN

$$
\dot{x}(t)=A_{6} x(t)+B_{6} u(t)+\omega(t), \quad y(t)=C_{6} x(t) .
$$

Rule 7: IF $x_{1}(t)$ is about $\pi / 2$ and $x_{2}$ is about $-\pi / 2$, THEN

$$
\dot{x}(t)=A_{7} x(t)+B_{7} u(t)+\omega(t), y(t)=C_{7} x(t) .
$$

Rule 8: IF $x_{1}(t)$ is about $\pi / 2$ and $x_{2}$ is about 0 , THEN

$$
\dot{x}(t)=A_{8} x(t)+B_{2} u(t)+\omega(t), \quad y(t)=C_{8} x(t) .
$$

Rule 9: IF $x_{1}(t)$ is about $\pi / 2$ and $x_{2}$ is about $\pi / 2$, THEN

$$
\dot{x}(t)=A_{9} x(t)+B_{9} u(t)+\omega(t), \quad y(t)=C_{9} x(t),
$$

where $x=\left[x_{1}, x_{2}, x_{3}, x_{4}\right]^{T}, u=\left[\tau_{1}, \tau_{2}\right]^{T}$. The parameter matrices $A_{i}, B_{i}, C_{i}$ are given in Appendix. It is supposed that the unknown disturbance $\omega(t)$ is bandlimited white noise with power 0.001 . It can be seen that $\operatorname{rank}(C B)=0$, so the proposed first estimation strategy cannot be designed in the robotic system.

The observer gains are given in Appendix by Theorem 3. We design the normal nonlinear controller based on backstepping control. The control objective is to force the system outputs $q_{1}$ and $q_{2}$ to track the sinusoidal desired trajectories $y_{1}^{d}=\sin (0.5 t)$ and $y_{2}^{d}=\sin (0.5 t)$. The parameters $k_{1}$ and $k_{2}$ of the controller can also be selected following Xu et al. (2011b).

We assumed that actuator faults are created as follows:

$$
\begin{aligned}
& \mu_{1}(t)= \begin{cases}0, & 0 \leq t<3 \\
50 \sin (2(t-3)), & 3 \leq t \leq 10\end{cases} \\
& \mu_{2}(t)= \begin{cases}0, & 0 \leq t<3 \\
50\left(1-e^{-4(t-3)}\right), & 3 \leq t \leq 10\end{cases}
\end{aligned}
$$

The system states are also estimated accurately as seen in Fig. 6. Figure 7 illustrates the result of actuator fault estimation. It can be seen that the proposed method is effective to estimate the actuator fault.
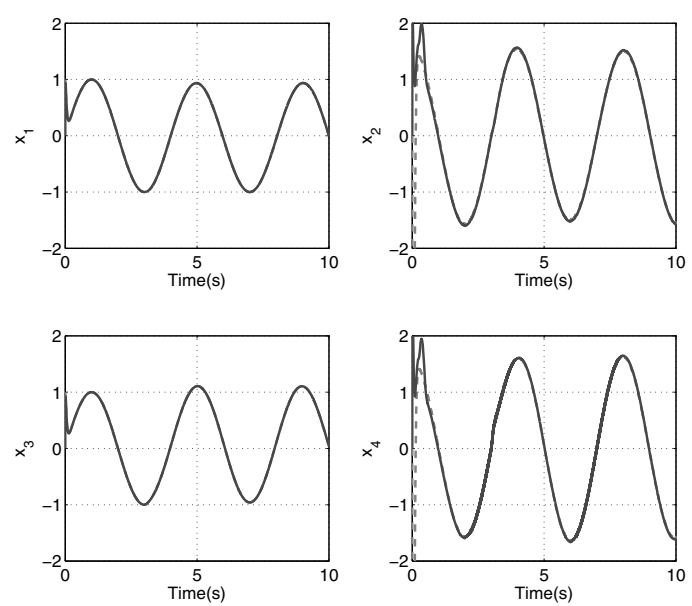

Fig. 6. Actual and estimated states (solid: actual, dotted: estimate).

The actuator fault and states can be estimated by the fault estimation observer without using adaptive and sliding mode technologies. The fault estimation observer can estimate a time-varying actuator fault quickly and the steady-state error for a constant actuator fault accurately.

\section{Conclusions}

This paper developed a methodology for actuator fault diagnosis and quantitative estimation of actuator fault signals in nonlinear systems via a T-S fuzzy model. The T-S 

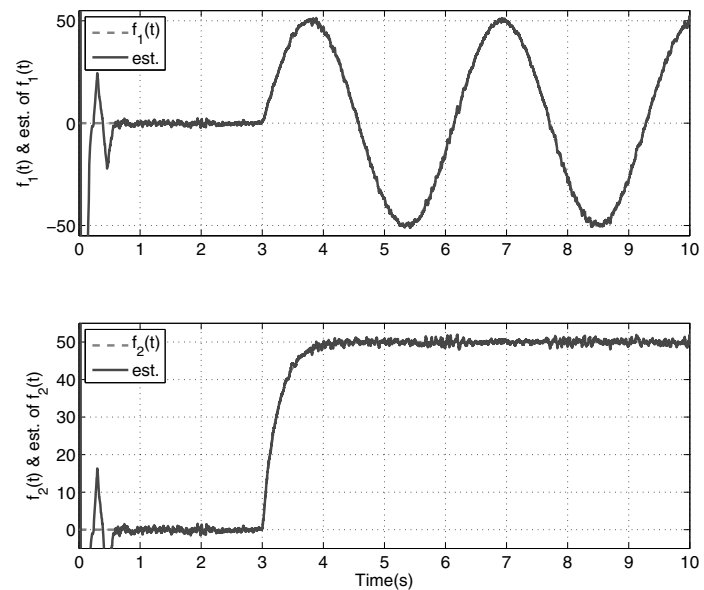

Fig. 7. Unknown fault input and its estimate.

fuzzy modeling approach is firstly employed to approximate the nonlinear dynamic system, and then the actuator fault model is presented. Two different actuator fault estimation strategies are developed. For the first strategy, a $\mathrm{T}-\mathrm{S}$ fuzzy observer was designed based on a T-S fuzzy model and the sliding mode technique, which is used to observe the state of the nonlinear system. Next, using the inverse system of the nonlinear system, the actuator fault can be estimated. The second strategy, combining a T-S fuzzy observer with an inverse system, is to estimate and observe the actuator fault and state. Simulation results are used to show the effectiveness of the obtained results. Actuator fault estimation system design for T-S fuzzy systems with an approximation error and application to practical nonlinear systems are meaningful and challenging issues, which will be studied in our future work.

\section{Acknowledgment}

The authors would like to thank the editors and reviewers for their very constructive comments and suggestions which have greatly helped improve the quality and presentation of the paper. This work was partially supported by the National Natural Science Foundation of China (61034005,61010121), the Funding of the Jiangsu Innovation Program for Graduate Education (CXZZ11-0213), and the UK Engineering and Physical Sciences Research Council (EP/F029195).

\section{References}

Babuska, R. (1998). Fuzzy Modeling for Control, Kluwer Academic Publishers, Boston, MA.

Boukezzoula, R., Galichet, S. and Folloy, L. (2003). Nonlinear internal model control: Application of inverse model based fuzzy control, IEEE Transactions on Fuzzy Systems 11(6): 814-829.

Boukezzoula, R., Galichet, S. and Foulloy, L. (2007). Fuzzy feedback linearizing controller and its equivalence with the fuzzy nonlinear internal model control structure, International Journal of Applied Mathematics and Computer Science 17(2): 233-248, DOI: 10.2478/v10006-007-0021-4.

Chang, C. and Yeh, Y. (2006). Variance constrained fuzzy control for observer-based T-S fuzzy models with minimizing auxiliary performance index, Journal of Intelligent and Fuzzy Systems 17(1): 59-69.

Chen, J. and Patton, R. (1999). Robust Model-Based Fault Diagnosis for Dynamic Systems, Kluwer, Boston, MA.

Chen, W. and Saif, M. (2010). Fuzzy nonlinear unknown input observer design with fault diagnosis applications, Journal of Vibration and Control 16(3): 377-401.

Christophe, C., Cocquempot, V. and Jiang, B. (2002). Link between highgain observer-based residual and parity space one, Proceedings of the American Control Conference, Anchorage, AK, USA, pp. 2100-2105.

Ding, X. and Frank, M. (1993). An adaptive observer-based fault detection schme for nonlinear systems, Proceedings of the 12th IFAC World Congress, Sydney, Australia, pp. 307312.

Edwards, C., Spurgeon, S. and Patton, R. (2000). Sliding mode observers for fault detection and isolation, Automatica 36(2): 541-553.

Fu, Y., Duan, G. and Song, S. (2004). Design of unknown input observer for linear time-delay systems, International Journal of Control, Automation, and Systems 2(4): 530-535.

Gao, H., Zhao, Y. and Chen, T. (2009). $\mathcal{H}_{\infty}$ fuzzy control of nonlinear systems under unreliable communication links, IEEE Transactions on Fuzzy Systems 17(2): 265-278.

Gao, Z., Jiang, B., Shi, P. and Xu, Y. (2010). Fault accommodation for near space vehicle attitude dynamics via T-S fuzzy models, International Journal of Innovative Computing Information and Control 6(11): 4843-4856.

Gu, Z., Peng, C. and Tian, E. (2010). Reliable control for a class of discrete-time state-delayed nonlinear systems with stochastic actuators failures, ICIC Express Letters pp. 2475-2480.

Guan, Y. and Saif, M. (1991). Novel approach to the design of unknown input observers, IEEE Transactions on Automatic Control 36(5): 632-635.

Guo, Y., Jiang, B. and Shi, P. (2010). Delay-dependent adaptive reconfiguration control in the presence of input saturation and actuator faults, International Journal of Innovative Computing, Information and Control 6(4): 1873-1882.

Isermann, R. (2005). Model-based fault detection and diagnosis status and application, Annual Reviews in Control 29(1): 71-85.

Isermann, R. (2006). Fault-Diagnosis Systems: An Introduction from Fault Detection to Fault Tolerance, Springer, Berlin. 
Jiang, B., Staroswiecki, M. and Cocquempot, V. (2001). Fault diagnosis for a class of nonlinear systems with unknown paramenters, Proceedings of the 4th IFAC Workshop on Online Fault Detection and Supervision in the Chemical Process Industries, Seoul, South Korea, pp. 181-186.

Jiang, B., Staroswiecki, M. and Cocquempot, V. (2006). Fault accommodation for nonlinear dynamic systems, IEEE Transactions on Automatic Control 51(9): 1805-1809.

Jiang, B., Zhang, K. and Shi, P. (2010). Less conservative criteria for fault accommodation of time-varying delay systems using adaptive fault diagnosis observer, International Journal of Adaptive Control and Signal Processing 24(4): 322334.

Kabore, R., Othman, S., Mckenna, T. and Hammouri, H. (2000). Observer-based fault diagnosis for a class of nonlinear systems-application to a free radical copolymerization reaction, International Journal of Control 73(9): 787-803.

Kabore, R. and Wang, H. (2001). Design of fault diagnosis filters and fault-tolerant control for a class of nonlinear systems, IEEE Transactions on Automatic Control 46(11): 18051810.

Lendek, Z., Guerra, T., Babuska, R. and Schutter, B. (2010a). Stability Analysis and Nonlinear Observer Design Using Takagi-Sugeno Fuzzy Models, Springer, Berlin.

Lendek, Z., Lauberb, J. and Guerra, T. (2010b). Adaptive observers for T-S fuzzy systems with unknown polynomial inputs, Fuzzy Sets and Systems 16(1): 2043-2065.

Nguang, S. and Shi, P. (2003). $\mathcal{H}_{\infty}$ fuzzy output feedback control design for nonlinear systems: An LMI approach, IEEE Transactions on Fuzzy Systems 11(3): 331-340.

Nguang, S., Shi, P. and Ding, X. (2007). Fault detection for uncertain fuzzy systems: An LMI approach, IEEE Transactions on Fuzzy Systems 15(6): 1251-1262.

Pang, H. and Tang, G. (2010). Global robust optimal sliding mode control for a class of nonlinear systems with uncertainties, ICIC Express Letters 4(6): 2501-2508.

Patton, R., Toribiot, C. and Simanit, S. (2001). Robust fault diagnosis in a chemical process using multiple-model approach, Proceedings of the 40th IEEE Conference on Decision and Control, Orlando, FL, USA, pp. 149-154.

Persis, C. and Isidori, A. (2001). A geometric approach to nonlinear fault detection and isolation, IEEE Transactions on Automatic Control 46(6): 853-865.

Polycarpou, M. (2001). Fault accommodation of a class of multivariable nonlinear dynamical systems using learing approach, IEEE Transactions on Automatic Control 46(5): 736-742.

Seliger, R. and Frank, M. (1991). Fault diagnosis by disturbance decoupled nonlinear observers, Proceedings of the 30th IEEE Control Decision Conference, Brighton, UK, pp. 2248-2253.

Shumsky, A. (2007). Redundancy relations for fault diagnosis in nonlinear uncertain systems, International Journal of Applied Mathematics and Computer Science 17(4): 477-489, DOI: 10.2478/v10006-007-0040-1.
Staroswiecki, M. and Gehin, A. (2001). From control to supervision, Annual Reviews in Control 25(1): 1-11.

Takagi, T. and Sugeno, M. (1985). Fuzzy identification of systems and its applications to modeling and control, IEEE Transactions on Systems, Man, and Cybernetics-Part B 17(2): 116-132.

Tanaka, K. and Wang, H. (2001). Fuzzy Control System Design and Analysis: A Linear Matrix Inequality Approach, John Wiley and Sons, New York, NY.

Vachtsevanos, G., Lewis, F. and Roemer, F. (2006). Intelligent Fault Diagnosis and Prognosis for Engineering Systems, John Wiley and Sons Ltd., Hoboken, NJ.

Wu, L., Su, X., Shi, P. and Qiu, J. (2011). Model approximation for discrete-time state-delay systems in the T-S fuzzy framework, IEEE Transactions on Fuzzy Systems 19(2): 366-378.

Xie, X., Zhou, D. and Jin, Y. (1999). Strong tracking filter based adaptive generic model control, Journal of Process Control 9(4): $337-350$.

Xu, Y., Jiang, B., Tao, G. and Gao, Z. (2011a). Fault accommodation for near space hypersonic vehicle with actuator fault, International Journal of Innovative Computing, Information and Control 7(5): 2187-2200.

Xu, Y., Jiang, B., Tao, G. and Gao, Z. (2011b). Fault tolerant control for a class of nonlinear systems with application to near space vehicle, Circuits, Systems, and Signal Processing 30(3): 655-672.

Yan, X. and Edwards, C. (2007). Nonlinear robust fault reconstruction and estimation using a sliding mode observer, $A u$ tomatica 43(9): 1605-1614.

Yang, Q. (2004). Model-based and Data Driven Fault Diagnosis Methods with Applications to Process Monitoring, Ph.D. thesis, Case Western Reserve University, Cleveland, $\mathrm{OH}$.

Zhang, K. and Jiang, B. (2010). Dynamic output feedback fault tolerant controller design for Takagi-Sugeno fuzzy systems with actuator faults, IEEE Transactions on Fuzzy Systems 18(1): 194-201.

Zhang, K., Jiang, B. and Shi, P. (2009). Fast fault estimation and accommodation for dynamical systems, IET Control Theory and Applications 3(2): 337-350.

Zhang, Y. and Jiang, J. (2008). Bibliographical review on reconfigurable fault-tolerant control systems, Annual Reviews in Control 32(1): 229-252.

Zhou, S., Lam, J. and Zheng, W. (2007). Control design for fuzzy systems based on relaxed nonquadratic stability and $\mathcal{H}_{\infty}$ performance conditions, IEEE Transactions on Fuzzy Systems 15(2): 188-199. 


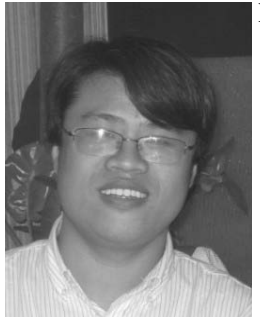

Dezhi $\mathrm{Xu}$ received a B.Sc. in automatic control from the North University of China, Taiyuan, in 2007, and an M.Sc. in automatic control from the Lanzhou University of Technology, Lanzhou, China, in 2010. He is currently a Ph.D. candidate with the College of Automation Engineering in the Nanjing University of Aeronautics and Astronautics. His research interests include fault diagnosis and fault-tolerant control for complex systems as well as data-driven

control.

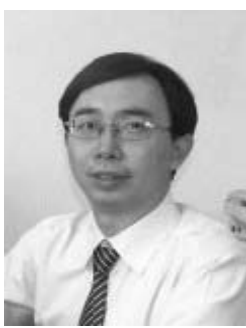

Bin Jiang received his $\mathrm{Ph} . \mathrm{D}$. degree in automatic control from Northeastern University, Shenyang, China, in 1995. He has been a postdoctoral fellow and a research fellow in Singapore, France and the USA. Now he is a professor and vice dean of the College of Automation Engineering in the Nanjing University of Aeronautics and Astronautics, China. He currently serves as an associate editor or editorial board member for several international journals. He is a senior member of the IEEE and a member of the IFAC Technical Committee on Fault Detection, Supervision, and Safety of Technical Processes. His research interests include fault diagnosis and fault tolerant control, and their applications.

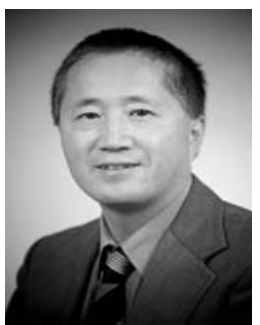

Peng Shi received the B.Sc. degree in mathematics from the Harbin Institute of Technology, China, in 1982, the M.E. degree in systems engineering from Harbin Engineering University in 1985, the Ph.D. degree in electrical engineering from the University of Newcastle, Australia, in 1994, and the Ph.D. degree in mathematics from the University of South Australia in 1998. He was awarded the D. Sc. degree by the University of Glamorgan, UK, in 2006. Dr Shi was a lecturer in Heilongjiang University, China (1985-1989), the University of South Australia (1997-1999), and a senior scientist in the Defence Science and Technology Organisation, Department of Defence, Australia (1999-2005). He joined in the University of Glamorgan as a professor in 2004. He has also been a professor at Victoria University, Australia, since 2008. Dr Shi's research interests include system and control theory, computational and intelligent systems, and operational research. He has published widely in these areas. Dr Shi currently serves as the editor-inchief of the International Journal of Innovative Computing, Information and Control and an associate editor for a number of journals. He is the recipient of the Most Cited Paper Award of Signal Processing in 2009. Dr Shi is a fellow of the Institute of Engineering and Technology (IET, UK), a fellow of the Institute of Mathematics and Its Applications (IMA, UK), and a senior member of the Institute of Electrical and Electronic Engineers (IEEE, USA).

\section{Appendix}

The parameter matrices $A_{i}, B_{i}, C_{i}$ are given as

$$
A_{1}=\left[\begin{array}{cccc}
0 & 0 & 1 & 0 \\
0 & 0 & 0 & 1 \\
5.927 & -0.315 & -0.001 & -8.4 \times 10^{-6} \\
-6.859 & 3.155 & 0.002 & 6.2 \times 10^{-6}
\end{array}\right]
$$

$$
A_{2}=\left[\begin{array}{cccc}
0 & 0 & 1 & 0 \\
0 & 0 & 0 & 1 \\
3.0482 & 0.1791 & -0.0011 & -0.0002 \\
3.5436 & 2.5611 & 0.0313 & 1.14 \times 10^{-5}
\end{array}\right],
$$$$
A_{3}=\left[\begin{array}{cccc}
0 & 0 & 1 & 0 \\
0 & 0 & 0 & 1 \\
6.2728 & 0.4339 & 0.0030 & -0.0001 \\
9.1041 & -1.0574 & 0.0158 & -3.2 \times 10^{-5}
\end{array}\right] \text {, }
$$

$$
A_{4}=\left[\begin{array}{cccc}
0 & 0 & 1 & 0 \\
0 & 0 & 0 & 1 \\
6.5435 & 1.2427 & 0.0017 & 0.0002 \\
-3.1873 & 5.1911 & -0.0306 & -1.8 \times 10^{-5}
\end{array}\right] \text {, }
$$$$
A_{5}=\left[\begin{array}{cccc}
0 & 0 & 1 & 0 \\
0 & 0 & 0 & 1 \\
11.1336 & -1.18145 & 0.0000 & 0.0000 \\
-9.0918 & 9.1638 & 0.0000 & 0.0000
\end{array}\right] \text {, }
$$

$$
A_{6}=\left[\begin{array}{cccc}
0 & 0 & 1 & 0 \\
0 & 0 & 0 & 1 \\
6.1702 & 1.6870 & -0.000 & 0.0002 \\
-2.3559 & 4.5298 & 0.0314 & 1.1 \times 10^{-5}
\end{array}\right] \text {, }
$$

$$
A_{7}=\left[\begin{array}{cccc}
0 & 0 & 1 & 0 \\
0 & 0 & 0 & 1 \\
6.1206 & 0.6205 & -0.0041 & 0.0001 \\
8.8794 & -1.0119 & -0.0193 & 4.4 \times 10^{-5}
\end{array}\right] \text {, }
$$

$$
A_{8}=\left[\begin{array}{cccc}
0 & 0 & 1 & 0 \\
0 & 0 & 0 & 1 \\
3.6421 & 0.0721 & 0.0018 & 0.0002 \\
2.4290 & 2.9832 & -0.0305 & -1.9 \times 10^{-5}
\end{array}\right] \text {, }
$$

$A_{9}=\left[\begin{array}{cccc}0 & 0 & 1 & 0 \\ 0 & 0 & 0 & 1 \\ 6.2933 & -0.2188 & -0.0009 & -1.2 \times 10^{-5} \\ -7.4649 & 3.2693 & 0.0024 & 9.2 \times 10^{-6}\end{array}\right]$.

$$
\begin{aligned}
& B_{1}=\left[\begin{array}{cc}
0 & 0 \\
0 & 0 \\
1 & -1 \\
-1 & 2
\end{array}\right], \quad B_{2}=\left[\begin{array}{cc}
0 & 0 \\
0 & 0 \\
0.5 & 0 \\
0 & 1
\end{array}\right], \\
& B_{3}=\left[\begin{array}{ll}
0 & 0 \\
0 & 0 \\
1 & 1 \\
1 & 2
\end{array}\right], \quad B_{4}=\left[\begin{array}{cc}
0 & 0 \\
0 & 0 \\
0.5 & 0 \\
0 & 1
\end{array}\right], \\
& B_{5}=\left[\begin{array}{cc}
0 & 0 \\
0 & 0 \\
1 & -1 \\
-1 & 2
\end{array}\right], \quad B_{6}=\left[\begin{array}{cc}
0 & 0 \\
0 & 0 \\
0.5 & 0 \\
0 & 1
\end{array}\right] \text {, } \\
& B_{7}=\left[\begin{array}{ll}
0 & 0 \\
0 & 0 \\
1 & 1 \\
1 & 2
\end{array}\right], \quad B_{8}=\left[\begin{array}{cc}
0 & 0 \\
0 & 0 \\
0.5 & 0 \\
0 & 1
\end{array}\right] \text {, }
\end{aligned}
$$




$$
B_{9}=\left[\begin{array}{cc}
0 & 0 \\
0 & 0 \\
1 & -1 \\
-1 & 2
\end{array}\right], \quad C_{i}=\left[\begin{array}{llll}
1 & 0 & 0 & 0 \\
0 & 1 & 0 & 0
\end{array}\right]
$$

The observer gains of Example 2 are given as

$$
\begin{aligned}
L_{1} & =\left[\begin{array}{cc}
4.5110 \times 10^{2} & -4.4693 \times 10 \\
-7.5851 \times 10 & 2.5735 \times 10^{2} \\
1.2168 \times 10^{3} & -1.7120 \times 10^{2} \\
-2.4621 \times 10^{2} & 7.7910 \times 10^{2}
\end{array}\right], \\
L_{2} & =\left[\begin{array}{cc}
4.6963 \times 10^{2} & -8.1664 \times 10 \\
-8.0699 \times 10 & 2.6313 \times 10^{2} \\
1.2667 \times 10^{3} & -2.7067 \times 10^{2} \\
-2.6021 \times 10^{2} & 7.9779 \times 10^{2}
\end{array}\right], \\
L_{3} & =\left[\begin{array}{cc}
4.0385 \times 10^{2} & -1.1052 \times 10^{2} \\
-6.4433 \times 10 & 2.7854 \times 10^{2} \\
1.0888 \times 10^{3} & -3.5054 \times 10^{2} \\
-2.0810 \times 10^{2} & 8.4484 \times 10^{2}
\end{array}\right], \\
L_{4} & =\left[\begin{array}{cc}
4.3523 \times 10^{2} & -5.5508 \times 10 \\
-8.8939 \times 10 & 2.5128 \times 10^{2} \\
1.1773 \times 10^{3} & -1.9855 \times 10^{2} \\
-2.8464 \times 10^{2} & 7.6144 \times 10^{2}
\end{array}\right],
\end{aligned}
$$

$$
L_{5}=\left[\begin{array}{cc}
3.7229 \times 10^{2} & -4.1800 \times 10 \\
-5.7890 \times 10 & 2.4382 \times 10^{2} \\
1.0041 \times 10^{3} & -1.6094 \times 10^{2} \\
-1.8940 \times 10^{2} & 7.3892 \times 10^{2}
\end{array}\right] \text {, }
$$$$
L_{6}=\left[\begin{array}{cc}
4.3828 \times 10^{2} & -5.9589 \times 10 \\
-9.2605 \times 10 & 2.5321 \times 10^{2} \\
1.1861 \times 10^{3} & -2.0975 \times 10^{2} \\
-2.9571 \times 10^{2} & 7.6731 \times 10^{2}
\end{array}\right] \text {, }
$$$$
L_{7}=\left[\begin{array}{cc}
4.0793 \times 10^{2} & -1.0870 \times 10^{2} \\
-6.6944 \times 10 & 2.7792 \times 10^{2} \\
1.1001 \times 10^{3} & -3.4554 \times 10^{2} \\
-2.1585 \times 10^{2} & 8.4292 \times 10^{2}
\end{array}\right] \text {, }
$$$$
L_{8}=\left[\begin{array}{cc}
4.6273 \times 10^{2} & -7.6872 \times 10 \\
-7.9199 \times 10 & 2.6137 \times 10^{2} \\
1.2481 \times 10^{3} & -2.5757 \times 10^{2} \\
-2.5558 \times 10^{2} & 7.9235 \times 10^{2}
\end{array}\right] \text {, }
$$$$
L_{9}=\left[\begin{array}{cc}
4.4698 \times 10^{2} & -4.2650 \times 10 \\
-7.6254 \times 10 & 2.5663 \times 10^{2} \\
1.2059 \times 10^{3} & -1.6560 \times 10^{2} \\
-2.4734 \times 10^{2} & 7.7684 \times 10^{2}
\end{array}\right] \text {. }
$$

Received: 13 January 2011 Revised: 15 July 2011

Re-revised: 7 November 2011 